\title{
Peertechz
}

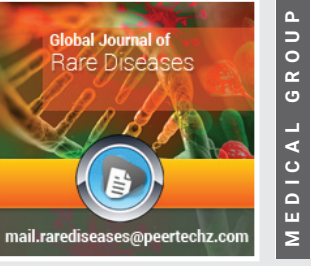

\section{Duane-Radial Ray syndrome a SALL4-Related Disorder. Report of a case in Chile}

\author{
Jonathan Huserman ${ }^{1 *}$ and Catherine Diaz ${ }^{1,2}$ \\ ${ }^{1}$ Hospital Clínico Universidad de Chile, Sección genética, Santiago, Chile \\ ${ }^{2}$ Hospital Roberto del Río, Sección genética, Santiago, Chile
}

Received: 24 August, 2020

Accepted: 05 September, 2020

Published: 07 September, 2020

*Corresponding author: Jonathan Huserman, Hospital Clinico Universidad de Chile, Seccion Genetica. Santiago, Chile, Email: jonathan.huserman@ug.uchile.cl ORCID: https://orcid.org/0000-0002-9355-3282

Keywords: Chile; SALL4; Duane-radial ray syndrome; Acrorenoocular syndrome; SALL4-related disorder

https://www.peertechz.com

Check for updates

\section{Abstract}

The Duane-Radial Ray syndrome or Okihiro syndrome belongs to the SALL4-Related Disorders, a phenotypic spectrum, that additionally includes, acrorenoocular syndrome and Holt-Oram syndrome, caused by the alteration of the same gene, which has significant relevance in the mesoderm, the limbs, and the heart development. These syndromes are characterized by thumb alteration, radial deviation of the forearm, Duane anomaly, and variable involvement of other organs such as kidney or heart. The prevalence is unknown. This is a case report of a Chilean patient with a pathogenic variant that confirms the Duane Radial Ray syndrome previously not described in the population.

\section{Abbreviations}

SALL4: Spalt like transcription factor 4; TBX5: T-Box Transcription Factor 5; LEF1: Lymphoid Enhancer Binding Factor; TCF: T cell transcription factor; FGF10: Fibroblast growth factor 10; TBX4: T-Box Transcription Factor 4; BMP4: Bone Morphogenetic Protein 4; CRBN: Cereblon; BAEP: Automated Brainstem Auditory Evoked Potential: BTE; Behind The Ear; ACMG: American College of Medical Genetics; IVIC: Venezuelan Institute for Scientific Research

\section{Introduction}

The Duane-Radial Ray syndrome or Okihiro syndrome (OMIM: 607323), belongs to the SALL4-related Disorders, a phenotypic spectrum, that also includes acrorenoocular and Holt-Oram Syndrome, previously considered to be different entities [1]. Duane-Radial Ray syndrome is characterized by radial aplasia or hypoplasia with shortening and radial deviation of the forearm, the thumb can be absent, partial absent, or triphalangeal with a postaxial polydactyly, and can be associated with Duane anomaly (Abduction ocular limitation due to abnormal VI cranial nerve innervation, presenting with ocular retraction in the abduction and a small palpebral fissure) $[1,2]$. On the other hand, the acrorenoocular Syndrome presents in addition to the dysmorphic features presented above, renal malformations (malrotation, ectopia, horseshoe kidney). Holt-Oram syndrome is characterized by a radial and heart malformations (atrial, ventricular septal defects, or Fallot tetralogy), with evidence of a pathogenic variant in SALL4 instead of a variant in $\mathrm{TBX}_{5}[1,3]$. The prevalence of these conditions is still unknown [1].

The SALL4 gene is part of the spalt-like gene family, transcription factors that possess a Zinc finger motif [4,5], mainly expressed during the fetal stage, decreasing their expression in adulthood, confined to the testis and ovaries $[5,6]$. It has significant importance in the maintenance of the cell multipotentiality and self-renewal [3]. This gene is located in 20q13.2, consisting of 4 exons with 2 possible isoforms generated by alternative splicing, both with the capacity to form homodimers [5]. The SALL4 protein acts by interacting with other transcription factors during embryonic development, such as SALL1, forming heterodimers that stimulate the canonical Wnt pathway, which in turn interacts with LEF1/TCF, increasing the expression of SALL4 in the heart and upper limb tissues. SALL4 also works together with TBX5 to regulate the development of the thumb, also together with FGF10, they determine the rest of the digits. In other tissues such as in the lower limbs bud, SALL4 interacts with TBX4, in cardiac tissue with $\mathrm{TBX}_{5}$, and in the mesoderm induces the expression of BMP-4 with interrelations with Wnt $[4,5,7]$. 
We present a case of a rare disease, with an unknown prevalence, not reported in Chilean population, with a clinical and molecular diagnosis of SALL4 related syndrome, with a novel pathogenic variant.

\section{Clinical presentation}

We present the case of a 2-years-1-month old patient, the second child of a healthy non-consanguineous couple of Peruvian origin. Uneventful pregnancy, and term delivery. After birth, an imperforate anus with perineal fistula, agenesis of the right thumb and bilateral radius, bilateral radial deviation of hand and right talipes equinovarus were observed (Figure 1A). Subsequently, polysyndactyly was detected between the first and second finger, bilateral camptodactyly, $5^{\text {th }}$ finger clinodactyly (Figure 1B), facial asymmetry, and small palpebral fissures.
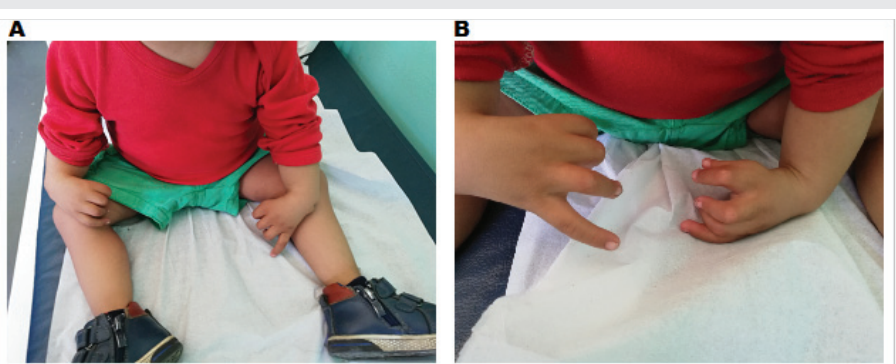

Figure 1: A. Shortening of the forearm, right thumb agenesis and actual state of lower limb with the use of splints can be observed. B. Absent of the right thumb, cutaneous syndactyly of the thumb and first finger of the left hand, can be observed.

Among the test performed, renal function was not assured with blood or urine test. The echocardiogram shows a patent ductus arteriosus, a patent foramen oval, and a minimal apical ventricular septal defect, all of them resolve spontaneously at 9 months. Radiography of the hand and bilateral forearm was requested, reporting bilateral radial hypoplasia, a larger rudimentary bone in the left forearm, and left preaxial polydactyly. Also, lumbosacral, renal, and bladder ultrasound, did not show any anomalies of kidney position or size. Brain and spinal cord MRI showed a Galassi type 2 arachnoid cyst in the left medial cranial fossa, supratentorial ventriculomegaly, focal thickening of the infundibular stem, and lipomatosis in filum terminale, without signs of the anchored cord are observed. BAEP with impedanciometry was requested, being altered, then BAEP was repeated, with evidence of severe hearing loss. An ophthalmological evaluation revealed a deficit in the abduction of the left eye consistent with Duane syndrome type 1, without alterations of the fundus. Finally, in the endocrinology evaluation, no alteration of the hormonal axes was detected.

Karyotype was requested resulting 46, XY. Due to suspicion of Duane-Radial Ray syndrome, a molecular study of the SALL4 gene was performed, using next-generation sequencing, which sowed a heterozygous pathogenic variant c.516_522del (p.Gly174Trpfs* 6) (NM_020436.3).
In that context a sagittal anorectosplasty was performed at 5 days after birth, with subsequent anal dilation, achieving good continence. Plaster and then lower limb orthoses were used for foot correction with a good response but maintained delayed gait. Orthotics and motor rehabilitation are performed for radial deviation hand. Given his severe hearing loss, bilateral pediatric BTE aids were indicated.

\section{Discussion}

According to the clinical characteristics of the proband, radial alterations, agenesis of the right thumb, left preaxial polydactyly, Duane anomaly, ulnar shortening, syndactyly of the first, and second finger, bilateral deviation of the hand, right talipes equinovarus, heart disease, hearing loss, imperforate anus, and facial asymmetry, made us suspecting of a disorder related to SALL4, specifically a Radial Ray Duane syndrome. Therefore, it was decided for sequence the gene, which resulted in a heterozygous pathogenic variant c.516_522del (p.Gly174Trpfs* 6), evidence a 7 bp deletion, which causes a change in the reading frame, generating a premature stop codon, leading to a nonsense-mediated mRNA decay[1,6], within the embryonic cells, affecting the translation of this gene, generating a smaller amount of the SALL4 protein, that has a fundamental role in the development of the limbs and other tissues such as the heart, mesodermal tissue, eye, and brain, explaining many of the symptoms.

The molecular study has been of substantial importance, in being able to relate different phenotypes such as acrorenoocular syndrome, Duane-Radial Ray syndrome, and some cases of Holt-Oram syndrome with a pathogenic variant in the SALL4 gene, demonstrating a continuous in phenotypes [1]. To date 20 pathogenic variants have been described, 19 of them generate a change in the reading frame and one affects the splicing site [8-10]. The variant of this patient c.516_522del is located in exon 2, although it is not reported on populations databases, it is classified as pathogenic, since the frameshift mechanism, described in genes with loss of heterozygosity, on the other had this variant is not found in population databases like gnomAD or DGV, and computational evidence infers a deleterious effect on this gene, according to the 2015 ACMG guidelines $[9,10]$. The new variant has been added in clinvar as 916548 [8].

Allelic heterogeneity has been described in the SALL4 gene, with the description of the IVIC syndrome (OMIM 147750) $[1,6]$, first described in 1980 in a Venezuelan family of 6 generations and 105 individuals [11], presenting an autosomal dominant syndrome of variable expressivity, with radial defects, extraocular muscle involvement, congenital hearing loss, imperforate anus, renal malrotation, thrombocytopenia, and leukocytosis, without presenting ocular retraction, the last tree symptoms, would differentiate this condition from the other syndromes of the spectrum of SALL4 Related Disorders $[1,6,11]$. Subsequently, in this group, a 1 base pair deletion was detected in exon 3 of the SALL4 gene that segregates with the alterations [6]. 4 affected families were described until 1997 with this syndrome [6]. Therefore, new studies are necessary to determine if they belong to the spectrum of disorders related to SALL4 or it is a different disorder [1].

Given the interaction of SALL4 protein, with other 
transcription factors, it is possible to observe the overlap between signs of other pathologies such as Holt-Oram syndrome caused by a variant of the TBX 5 gene, characterized by malformations of the upper limbs (clinodactyly, thumb agenesis, or first metacarpal alteration and malformation of the carpus) and heart (atrial or ventricular septal defect) [3]. It is also similar to Townes-Brocks syndrome, secondary to a SALL1 gene variant, presenting the triad of the dysplastic ear, imperforate anus, and preaxial polydactyly or triphalangeal thumb, besides, they can present hearing loss, malformation of the feet, alteration of renal function or structure, genitourinary malformations and congenital heart disease [12]. On the other hand, Fanconi anemia caused by mutations in DNA repair genes may also present radial abnormalities and other signs, such as skeletal disorders, birth defects, urogenital abnormalities, hypogonadism, hypoacusis, imperforate anus, psychomotor development delay, but they differ in that in addition to anemia there are alterations of leukocytes and absence of Duane anomaly $[1,13]$. Another differential diagnosis is thalidomide embryopathy, a sedative, non-addictive, nontoxic, teratogenic drug, creating a spectrum of effects from the triphalangeal thumb, radial dysplasia (absence of radio and thumb), phocomelia or amelia [14]. One of the proposed mechanisms is that cereblon (CRBN), an ubiquitin ligase, binds to thalidomide, then recruits SALL4, inhibiting its signaling pathway and post-transcriptional degradation of this protein $[14,15]$.

The management of these patients must be multidisciplinary and personalized. In the case of Duane anomaly, the use of glasses to avoid vices of refraction, management of amblyopia, or even surgery of external ocular muscles according to severity [2]. The use of orthotics, rehabilitation, and surgery on the upper limbs is indicated according to the degree of deformity and functionality [1]. The correction of heart disease is carried out with conventional medical and surgical treatment. Given the possible existence of renal alteration medical or surgical management is prudent according to the anomalies it presents. Regarding severe hearing loss, the use of hearing aids or cochlear implants is recommended. For growth hormone deficiency, it must be supplemented to obtain the best height possible [1]. The long-term prognosis is not known, given the low number of patients described. So, it requires more study and follows up.

The genetic counseling, since it is inherited an autosomal dominant way, the children of the proband will have a $50 \%$ chance of inheriting the mutated gene, while the risk of the brothers of the proband will depend on whether they have an affected parent or not if any of them are affected there is a $50 \%$ chance to inherit the variant, while if the parents are healthy the risk of the brothers is $1-5 \%$ due to germinal mosaicism [1]. In our case, however, we did not have a molecular study of the parents, since none of them presented characteristics compatible with the clinical presentation, it could be considered a de novo pathogenic variant of the patient.

\section{Conclusion}

In conclusion, this is a case report on a Chilean patient diagnosed with a SALL4-Related syndrome, with confirmation of a pathogenic variant, not previously described in the population. The description of the phenotype was particularly important to have a high index of clinical suspicion. With the above, we managed to discover the etiology of the clinical presentation, providing proper management and multidisciplinary rehabilitation.

\section{Ethical considerations}

Both parents gave consent to show clinical data and photographs and gave permission to this report.

\section{References}

1. Adam MP, Ardinger HH, Pagon RA (2015) SALL4-Related Disorders.

2. Adam MP, Ardinger HH, Pagon RA (2019) Duane Syndrome Synonyms: Duane Anomaly, Isolated; Duane Retraction Syndrome; Stilling-Turk-Duane Syndrome.

3. Li B, Chen S, Sun K, Xu R, Wu Y (2018) Genetic Analyses Identified a SALL4 Gene Mutation Associated with Holt-Oram Syndrome. DNA Cell Biol 37: 398 404. Link: https://bit.ly/3INeOwh

4. Alves LU, Perez ABA, Alonso LG, Otto PA, Mingroni-Netto RC (2016) Nove frameshift variant in gene SALL4 causing Okihiro syndrome. Eur J Med Genet 59: 80-85. Link: https://bit.ly/2F42FHr

5. Tatetsu H, Kong NR, Chong G, Amabile G, Tenen DG, et al. (2016) SALL4, the missing link between stem cells, development and cancer HHS Public Access. Gene 584: 111-119. Link: https://bit.ly/3i74lyg

6. Paradisi I, Arias S (2007) IVIC syndrome is caused by a c.2607delA mutation in the SALL4 locus. Am J Med Genet Part A 143: 326-332. Link: https://bit.ly/2Z6ay5V

7. Al-Qattan MM (2011) Wnt pathways and upper limb anomalies. J Hand Surg Eur 36: 9-22. Link: https://bit.ly/3IPvCYi

8. National Center for Biotechnology Information. ClinVar, "[VCV000916548.2] Link: https://bit.ly/3217xWA

9. “NM_020436.5(SALL4):c.516_522del Deletion (7bp) | hg19.” [Online]. Link: Link: https://bit.ly/3hXxxaS

10. Kopanos C, Tsiolkas V, Kouris A, Chapple CE, Aguilera MA, et al. (2019) VarSome: the human genomic variant search engine. Bioinformatics 35 1978-1980. Link: https://bit.ly/3jREivE

11. Arias S, Penchaszadeh VB, Pinto-Cisternas J, Larrauri S (1980) The IVIC syndrome: A new autosomal dominant complex pleiotropic syndrome with radial ray hypoplasia, hearing impairment, external ophthalmoplegia, and thrombocytopenia. Am J Med Genet 6: 25-29. Link: https://bit.ly/3jMVOfu

12. Adam MP, Ardinger HH, Pagon RA (2015) Townes-Brocks Syndrome.

13. Adam MP, HH A, Pagon RA (2018) Fanconi anemia.

14. Vargesson $N$ (2019) The teratogenic effects of thalidomide on limbs. Journa of Hand Surgery European 44: 88-95. Link: https://bit.ly/357PeAR

15. do J, Gomes A, Kowalski TW, Fraga LR, Macedo GS, et al. (2019) The role of ESCO2, SALL4 and TBX5 genes in the susceptibility to thalidomide teratogenesis. Sci Rep 9: 1-11. Link: https://go.nature.com/3i4NAUl

Copyright: ( $) 2020$ Huserman J, et al. This is an open-access article distributed under the terms of the Creative Commons Attribution License, which permits unrestricted use, distribution, and reproduction in any medium, provided the original author and source are credited. 